\title{
More nitrogen rich B-type stars in the SMC cluster, NGC 330
}

\author{
D. J. Lennon ${ }^{1}$, P. L. Dufton ${ }^{2}$, and C. Crowley ${ }^{1}$ \\ ${ }^{1}$ Isaac Newton Group of Telescopes, Apartado de Correos 321, 38700 Santa Cruz de La Palma, Canary Islands, Spain \\ 2 The Department of Pure and Applied Physics, The Queen's University of Belfast, Belfast BT7 1NN, N. Ireland \\ e-mail: P.Dufton@Queens-Belfast.ac.uk
}

Received 12 June 2002 / Accepted 9 August 2002

\begin{abstract}
High resolution spectra of seven early B-type giant/supergiant stars in the SMC cluster NGC 330 are analysed to obtain their chemical compositions relative to SMC field and Galactic B-type stars. It is found that all seven stars are nitrogen rich with an abundance approximately 1.3 dex higher than an SMC main-sequence field B-type star, AV304. They also display evidence for deficiencies in carbon, but other metals have abundances typical of the SMC. Given the number of B-type stars with low projected rotational velocities in NGC 330 (all our targets have $v \sin i<50 \mathrm{~km} \mathrm{~s}^{-1}$ ), we suggest that it is unlikely that the stars in our sample are seen almost pole-on, but rather that they are intrinsically slow rotators. Furthermore, none of our objects displays any evidence of significant Balmer emission excluding the possibility that these are Be stars observed pole-on. Comparing these results with the predictions of stellar evolution models including the effects of rotationally induced mixing, we conclude that while the abundance patterns may indeed be reproduced by these models, serious discrepancies exist. Most importantly, models including the effects of initially large rotational velocities do not reproduce the observed range of effective temperatures of our sample, nor the currently observed rotational velocities. Binary models may be able to produce stars in the observed temperature range but again may be incapable of producing suitable analogues with low rotational velocities. We also discuss the clear need for stellar evolution calculations employing the correct chemical mix of carbon, nitrogen and oxygen for the SMC.
\end{abstract}

Key words. stars: early-type - supergiants - rotation - evolution

\section{Introduction}

NGC 330 is one of the brightest and most populous young clusters in the Small Magellanic Cloud (SMC). The photometric surveys of Arp (1959), Robertson (1974) and Carney et al. (1985) illustrate the key features of the cluster's colourmagnitude diagram, namely the presence of two groups of blue and red supergiants well separated from the cluster's supposed main-sequence blue plume. These two groups of stars have been widely interpreted as core helium burning stars and the cluster is therefore considered as a key test of stellar evolution theory and physics for stars of intermediate mass in a low metallicity regime. Essentially the ratio of blue (B) to red (R) supergiants is an indicator of the relative times a massive star spends in these phases, and these quantities are extremely sensitive to assumptions made concerning convection and mixing. In fact the $\mathrm{B} / \mathrm{R}$ ratio in NGC 330 is generally assumed to be representative of the SMC as a whole and is used as a calibrator for stellar evolution calculations at low metallicity (Stothers \& Chin 1992a, 1992b; Keller et al. 2000; Chiosi et al. 1995). The specific problem of the $\mathrm{B} / \mathrm{R}$ ratio as a function of metallicity has been discussed by Langer \& Maeder (1995), where a more

Send offprint requests to: D. J. Lennon, e-mail: dj1@ing.iac.es detailed discussion of the various treatments of convection and overshooting may be found.

The interpretation of the cluster's HR diagram is complicated by the surprise finding that many main sequence B-type stars in the cluster have $\mathrm{H} \alpha$ emission implying a very high incidence of Be stars (Feast 1972). A subsequent intermediate band and $\mathrm{H} \alpha$ photometric study indicated that at least $60 \%$ of all main-sequence B-type stars are of Be-type (Grebel et al. 1996), this high fraction being confirmed independently by the spectroscopic observations of Lennon et al. (1993), Mazzali et al. (1996) and Keller \& Bessell (1998). As with the ratio of B/R supergiants, the ratio Be/B-type stars in NGC 330 is often taken as being representive for the SMC metallicity (Maeder et al. 1999). A second complication arises concerning uncertainty over the metallicity of stars in NGC 330; some estimates of the metallicity based upon spectroscopy of the brightest $\mathrm{K}$ and F-type supergiants (Spite et al. 1991) and one B-type giant (Reitermann et al. 1990) imply that these objects are metal poor even with respect to SMC field stars, while Strömgren photometric observations of supergiants by Grebel \& Richtler (1992) have been interpreted as evidence for a metal deficiency of 0.5 dex with respect to field stars. However more recent analyses of K-type supergiants have tended to suggest that this difference in metallicity is much smaller, or indeed not significant 
(Hill 1999), confirming the results obtained from the analysis of two B-type stars in the cluster by Lennon et al. (1996, hereafter Paper I).

The spectroscopic work of Lennon et al. (1993) also found that the bright non-Be and weak Be-type stars occupied that region of the HR-diagram known as the post main sequence gap, or blue Hertzsprung gap (BHG). That is, they are giant/supergiant stars lying red-wards of the main sequence band, but blue-wards of the A/F-type supergiant regime. Caloi et al. (1993) and Grebel et al. (1996) have also commented on this fact, the latter suggesting that these stars are most likely a mixture of rapidly rotating $\mathrm{B} / \mathrm{Be}$-type stars of varying orientation and blue stragglers formed by interaction in binary stars. Keller et al. (2000) also attempted to address this problem using far-UV photometry (from the F160BW filter on WFPC2 of HST) to constrain B-type stellar effective temperatures and find significantly fewer stars in the blue Hertzsprung gap (BHG). However they assumed that the logarithmic surface gravities were 4.0 in their work (Keller, private communication), which may result in spuriously high effective temperatures for stars near the turn-off since they have much lower surface gravities (Lennon et al. 1993). Note that Caloi et al. (1993) also adopted lower values for the surface gravities. Clearly a detailed spectroscopic analysis of the BHG stars leading to estimates of both stellar parameters and atmospheric abundances is needed for comparison with the predictions of various stellar evolution calculations.

In Paper I, we derived metallicities of two such B-type stars in NGC 330 and while we found them in general to be compatible with that of the SMC field both stars had a significant nitrogen overabundance. The magnitude of the nitrogen enrichment was uncertain due to the small number of NGC 330 targets analysed and also the difficulty in estimating the low nitrogen abundance of the SMC field, at least, from B-type stars. Also in Paper I we found that the carbon abundance was not significantly depleted, contrary to what one expects if the nitrogen were produced in the $\mathrm{CN}$ cycle. The carbon abundance was uncertain however, and coupled with the uncertainty of the magnitude of the nitrogen enrichment, made interpretation difficult. An additional puzzling aspect was that both stars are narrow-lined and therefore if the nitrogen enhancements are the result of high rotation we must be observing them almost pole-on, which seemed unlikely give that they were drawn from the sample of about 20 stars observed by Lennon et al. (1993). In the present paper we analyse seven targets in NGC 330 (including the two discussed in Paper I) belonging to both the blue supergiant group and the tip of the blue main sequence plume discussed above. Hence all stars lie in, or close to, the BHG. We estimate stellar parameters, radial and projected rotational velocities, as well as both absolute abundances and differential abundances relative to a galactic target in the $\mathrm{h}$ and $\chi$ Persei cluster (Vrancken et al. 2000) and to an SMC field star, AV304. For the latter we utilise the results from a recent analysis (Rolleston et al 2002) based on high quality VLT data, which now give us a reliable estimate of the pristine nitrogen abundance in unevolved B-type stars in the SMC. We also attempt to provide improved estimates for carbon abundances and compare our results with stellar evolution calculations, including the recent models of Maeder \& Meynet (2001) which include the effects of rotationally induced mixing.

\section{Observational data and results}

\subsection{Optical spectroscopy}

High resolution spectra of early-type stars in the cluster NGC 330, were obtained using the CASPEC spectrograph on the ESO $3.6 \mathrm{~m}$ on the 26 and 27 August 1994 and on the 22 and 23 September 1996; spectral coverage was 3959-5147 and other observational parameters are summarized in Table 1. The $U B V$ photometry has been taken from Mazzali et al. (1996) supplemented by additional unpublished data, while the spectral types have been estimated from unpublished low dispersion EMMI spectroscopy. The observational configuration and data reduction techniques are discussed in detail in Paper I. Briefly, Caspec was used with an entrance slit width of 2 arcsec giving an effective resolving power of approximately 20000 , or about $15 \mathrm{~km} \mathrm{~s}^{-1}$. Preliminary reduction of the echellograms to a one dimensional format was achieved using the IRAF reduction package (Willmarth \& Barnes 1994). The relative faintness of the targets, lead to some of the spectra having relatively low continuum counts and signal-to-noise ratios. In particular, the observations for A01, B04 and B32 were taken in conditions that were at times cloudy, and so not all spectra were co-added to produce the final spectrum for these stars. The $s / n$ estimates are summarized in Table 1 and were measured near the centre of the echelle order at approximately $4200 \AA$. Fortunately, all the stars have small projected rotational velocities and are hence sharp lined with line widths ( $F W H M$ ) ranging from 0.4 to $0.9 \AA$ (see Table 1). Hence despite the significant shot noise, lines with equivalent widths of more than $50 \mathrm{~m} \AA$ could normally be detected, while features with line strengths as small as $30 \mathrm{~m} \AA$ could sometimes be convincingly identified. In Fig. 1, sample normalized spectra are shown for all the NGC 330 stars and for the SMC main sequence star AV 304. The latter has been taken from Rolleston et al. (2002) but has been rebinned and convolved with a Gaussian filter so that its line widths are comparable to those of the NGC 330 targets. Two spectral regions are shown covering the $\mathrm{N}_{\text {II }}$ line at $3995 \AA$ and the $\mathrm{O}_{\text {II }}$ multiplet near $4070 \AA$. Although the line strengths vary due to the different atmospheric parameters of the targets, it is clear that the $\mathrm{N}_{\text {II }}$ line is enhanced in all the NGC 330 targets compared to that in AV 304. Projected rotational velocities were estimated from the line widths by assuming that the only contributions to the intrinsic widths of the absorption lines are instrumental and pure rotational i.e. that the effects of macroturbulence are negligible. Hence the results in Table 1 are most likely upper limits on the projected rotational velocities.

Equivalent widths were measured for the metal and nondiffuse helium lines using the STARLINK spectrum analysis program DIPso. Absorption lines were fitted using a non-linear least squares technique with a variety of profile shapes (e.g. triangles, Gaussians) and degrees of polynomial for the continuum being considered. Equivalent widths for the diffuse helium lines were measured manually with the continuum being arbitrarily defined at $\pm 10 \AA$ from the line centre. For the hydrogen 

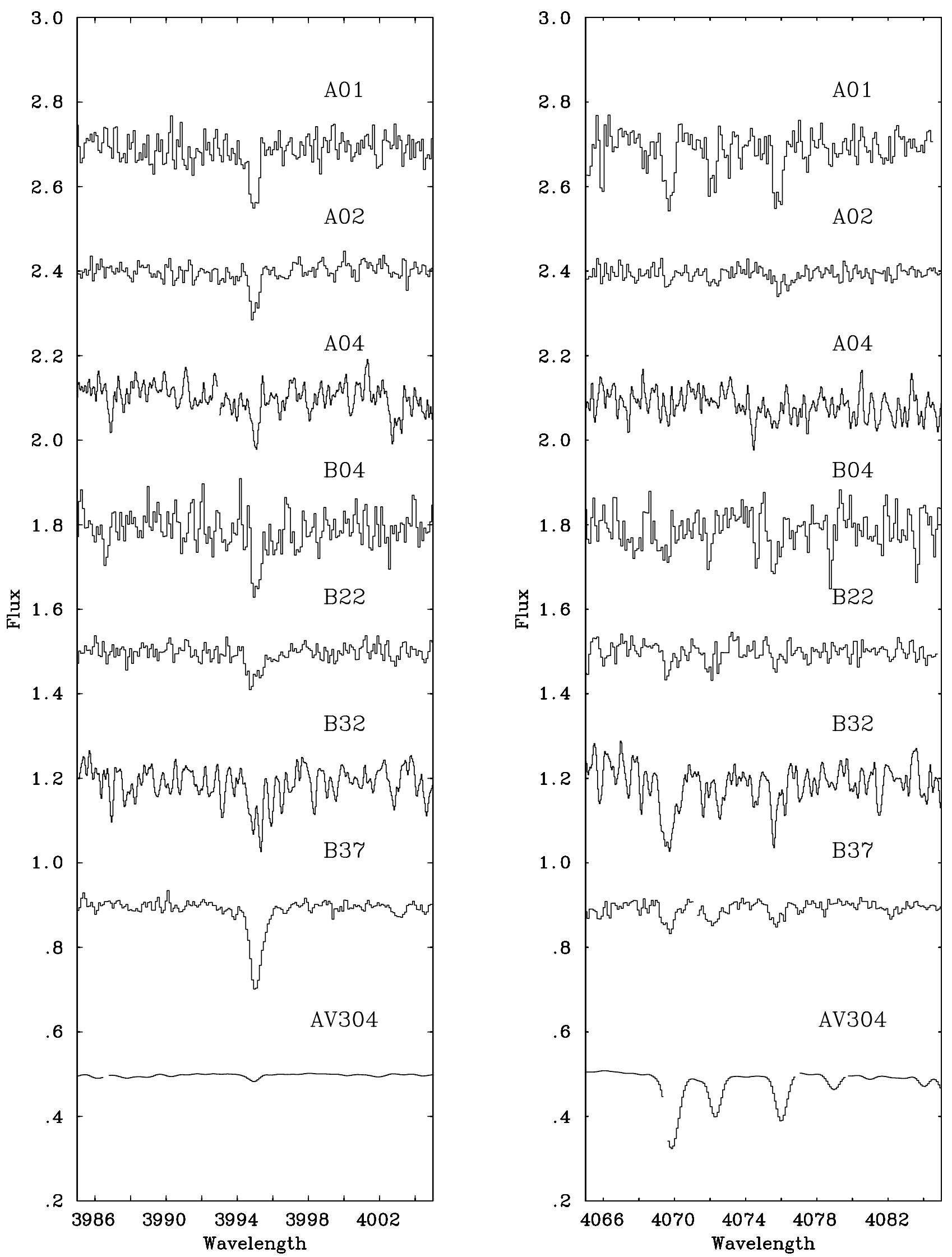

Fig. 1. Sample normalized spectra are shown for all the NGC 330 stars and for the SMC main sequence field star AV 304 . The latter has been taken from Rolleston et al. (2002) but has been rebinned and convolved with a Gaussian filter so that its line widths are comparable with those of the NGC 330 targets. Two spectral regions are shown covering the $\mathrm{N}_{\text {II }}$ line at $3995 \AA$ and the O II mutiplet near $4070 \AA$. 
Table 1. Observational data for NGC 330 targets: star identifications are from Robertson (1974). Spectral types are from Lennon et al. (1993) or Grebel et al. (1996) although some classifications are slightly revised. Photometry is taken mostly from Mazzali et al. (1996) with additional data taken from Grebel et al. (1996) and Robertson (1974). As discussed in the text, the projected rotational velocities $(v \sin i)$ are most probably upper limits, and are accurate to $\pm 5 \mathrm{~km} \mathrm{~s}^{-1}$. Radial velocities $\left(v_{\mathrm{rad}}\right)$ are corrected to heliocentric values.

\begin{tabular}{|c|c|c|c|c|c|c|c|c|c|c|}
\hline Star & Sp. Type & $\bar{V}$ & $B-V$ & $U-B$ & $\begin{array}{r}\text { Date } \\
\text { yymmdd }\end{array}$ & $\begin{array}{r}\text { Exposures } \\
n \times \mathrm{s} \\
\end{array}$ & $s / n$ & $\begin{array}{c}F W H M \\
\AA\end{array}$ & $\begin{array}{c}v \sin i \\
\mathrm{~km} \mathrm{~s}^{-1}\end{array}$ & $\begin{array}{c}v_{\text {rad }} \\
\mathrm{km} \mathrm{s}^{-1}\end{array}$ \\
\hline A01 & B0.5 III/V & 14.70 & -0.18 & -0.85 & 940828 & $3 \times 3600$ & 40 & 0.6 & 30 & $+151 \pm 6$ \\
\hline A02 & B4 Ib & 12.90 & -0.05 & -0.69 & 940828 & $\begin{array}{r}2 \times 1200 \\
1 \times 600\end{array}$ & 69 & 0.6 & 30 & $+151 \pm 3$ \\
\hline A04 & B2-3 IIe & 14.54 & -0.03 & - & 960923 & $2 \times 3000$ & 27 & 0.4 & 20 & $+155 \pm 5$ \\
\hline B04 & B2 III & 15.60 & -0.16 & -0.82 & 940827 & $\begin{array}{l}3 \times 3600 \\
2 \times 1800\end{array}$ & 25 & 0.4 & 20 & $+150 \pm 4$ \\
\hline B22 & B2 II & 14.29 & -0.13 & -0.75 & 940828 & $\begin{array}{l}1 \times 3600 \\
1 \times 2100\end{array}$ & 51 & 0.8 & 35 & $+157 \pm 4$ \\
\hline B32 & B2 III & 15.01 & -0.17 & - & $\begin{array}{l}960923 \\
960924\end{array}$ & $\begin{array}{l}2 \times 3600 \\
2 \times 3600\end{array}$ & 22 & 0.9 & 40 & $+157 \pm 7$ \\
\hline B37 & B3 Ib & 13.19 & -0.07 & -0.80 & 940827 & $2 \times 1800$ & 90 & 0.8 & 35 & $+156 \pm 3$ \\
\hline
\end{tabular}

$\mathrm{H} \gamma$ and $\mathrm{H} \delta$ lines, the spectra were again normalised and profiles measured, with the continuum levels now being defined at $\pm 16 \AA$ (as were the theoretical profiles). For all these measurements the procedures were effectively identical to those described in Paper I where further details can be found. Metal line equivalent widths are listed in Table 2.

The model atmosphere analysis utilised both absolute and differential techniques. For the latter, two standard stars were considered BD +56576 in the $\mathrm{h}$ and $\chi$ Persei galactic cluster and the SMC target AV304. The observational data for BD +56576 was taken from our study (Vrancken et al. 2000) of early-B type stars in this double cluster, while the results for AV304 are based on recently obtained VLT spectra (Rolleston et al. 2002).

\subsection{Flux distributions}

IUE low resolution spectra are available in both the short and long wavelength cameras for all the NGC 330 targets (apart from A04) and for BD +56576 . These were extracted from the INES archive (Rodriguez-Pascuel et al. 1999), where further details may be found.

\section{Method of analysis}

We initially consider standard LTE model atmosphere techniques in our analysis, however in Sect. 5 we discuss the accuracy of this method and correct our abundances for NLTE effects. Here we use the model atmospheres taken from the grids calculated with the code of Kurucz (1992) and made available at his website. This grid covers a range of chemical compositions, with the current analysis utilising mainly the results for metallicities of -0.5 dex, which is compatible with that found in Paper I. Details of the methods and atomic data used in the radiative transfer calculations can be found in, for example, Rolleston et al. (2000) or Smartt et al. (1996).

\subsection{Atmospheric parameters}

As discussed in Paper I, the relatively low signal-to-noise of the Caspec spectra preclude the observation of different ionization stages of the same element for most of the stars. Additionally there is no extant Strömgren photometry. Hence none of the standard techniques (see, for example, Kilian 1994; Gies \& Lambert 1992; Rolleston et al. 2000) are available for constraining effective temperatures. Hence, initial temperature estimates were made from the observed flux distributions. The extinction toward NGC 330 has been found to be relatively small and here we have adopted a foreground reddening of $E(B-V)=0.034$ and the extinction relation of Seaton (1979), together with an SMC extinction of $E(B-V)=0.05$ and the relationship of Thompson et al. (1988). As discussed in Paper I, similar effective temperatures would have been estimated using, for example, the galactic extinction law for all the reddening.

The effective temperature estimates are listed in Table 3. As discussed in Paper I, at effective temperatures greater than approximately $22000 \mathrm{~K}$, the flux distribution becomes relatively insensitive to this parameter. Additionally there may be uncertainties due to the presence of fainter targets in the IUE aperture. Hence we adopt conservative error estimates of $2000 \mathrm{~K}$ for our cooler targets and up to $4000 \mathrm{~K}$ for our hottest targets. For the latter, we note (see Paper I for details) that our estimates are consistent with the absence of a detectable He II spectrum, which implies that the effective temperatures must be less than $26000 \mathrm{~K}$. 
Table 2. Equivalent widths (mA) for the programme stars. Typical uncertainties lie in the range $10-20 \mathrm{~m} \AA$.

\begin{tabular}{|c|c|c|c|c|c|c|c|}
\hline Line & A01 & A02 & A04 & B04 & B22 & B32 & B37 \\
\hline \multicolumn{8}{|l|}{$\mathrm{C}_{\mathrm{II}}$} \\
\hline 3920 & - & 36 & 71 & - & 46 & - & 28 \\
\hline 4074.13 & - & - & - & - & 27 & - & - \\
\hline 4267 & 76 & 68 & 57 & 89 & 90 & 50 & 67 \\
\hline 4372.49 & - & 15 & - & - & - & - & - \\
\hline \multicolumn{8}{|l|}{$\mathrm{N}_{\text {II }}$} \\
\hline 3995.00 & 93 & 60 & 46 & 88 & 75 & 63 & 140 \\
\hline 4035.09 & 48 & - & - & 60 & 37 & - & 32 \\
\hline 4041.32 & 47 & 25 & - & 47 & - & - & 29 \\
\hline 4043.53 & 45 & 22 & - & 43 & 32 & - & 39 \\
\hline 4237.04 & 23 & 15 & - & 18 & - & - & - \\
\hline 4447.03 & 51 & 25 & - & 44 & 47 & - & 59 \\
\hline 4630.54 & 55 & 42 & 23 & 36 & 57 & 57 & 107 \\
\hline 5001.46 & 66 & 23 & - & - & 60 & 52 & 112 \\
\hline 5005.14 & - & 46 & - & - & 57 & - & 74 \\
\hline 5045.09 & - & 25 & - & - & - & - & 66 \\
\hline \multicolumn{8}{|l|}{$\mathrm{O}_{\text {II }}$} \\
\hline 4069.8 & 82 & 27 & 34 & 65 & 40 & 105 & 55 \\
\hline 4072.16 & 57 & 19 & - & 26 & - & - & 35 \\
\hline 4075.86 & 75 & 21 & - & 69 & 39 & 67 & 42 \\
\hline 4317.14 & 42 & 15 & - & - & 28 & - & 22 \\
\hline 4319.63 & 48 & 17 & - & - & 20 & - & 24 \\
\hline 4349.43 & 77 & 25 & 36 & - & - & 76 & 41 \\
\hline 4351.27 & 57 & 15 & - & - & 13 & - & - \\
\hline 4366.90 & 46 & 23 & - & - & - & - & 34 \\
\hline 4414.90 & 68 & - & - & 53 & - & - & - \\
\hline 4416.97 & 51 & 17 & - & 34 & 42 & - & 30 \\
\hline 4590.97 & 50 & - & - & - & - & - & - \\
\hline 4596.17 & 51 & - & - & - & - & - & 21 \\
\hline 4638.85 & 39 & - & - & - & 31 & - & - \\
\hline 4641.81 & 62 & 16 & - & - & - & - & 41 \\
\hline 4649.14 & 65 & 22 & - & - & - & - & 67 \\
\hline 4651.35 & 33 & 10 & - & - & - & - & 21 \\
\hline \multicolumn{8}{|l|}{$\mathrm{Mg}_{\text {II }}$} \\
\hline 4481 & 73 & 155 & 88 & 71 & 86 & - & 127 \\
\hline \multicolumn{8}{|l|}{ Si II } \\
\hline 4128.05 & - & 65 & 31 & - & - & - & - \\
\hline 4130.88 & - & 86 & 27 & - & - & - & - \\
\hline \multicolumn{8}{|l|}{ Si IIII } \\
\hline 4552.62 & 103 & 60 & 31 & 105 & 65 & 66 & 162 \\
\hline 4567.82 & 95 & 44 & 18 & 38 & 66 & - & 97 \\
\hline 4574.76 & 32 & - & - & 35 & 51 & - & 69 \\
\hline \multicolumn{8}{|l|}{ Si IV } \\
\hline 4116.2 & 27 & - & - & - & - & - & \\
\hline
\end{tabular}

Two stars, A01 and B04, had been already been discussed in Paper I; here we have independently re-estimated their effective temperatures. For the latter the two estimates are consistent, whilst for A01, the current estimate is $2000 \mathrm{~K}$ less than in Paper I. Here, we used flux distributions calculated for a metallicity 0.5 dex less than solar (in Paper I, fluxes for a normal metallicity were adopted) but tests showed that the estimates were little affected by the choice of metallicity; hence we ascribe the difference mainly to the flux distributions not being particularly temperature sensitive in the case of A01.

For A04, no IUE observations were available; there are, however, measurements of the equivalent widths of both Si III and Si Iv ions and these have been used to estimate an effective temperature (see, for example, Rolleston et al. 2000 for details) and this is also listed in Table 3. Note that the equivalent widths are small (typically $30 \mathrm{m \AA}$ ) and hence subject to considerable uncertainty. Additionally several authors (for example, Kilian 1994; Smartt et al. 2001) have found systematic differences between temperatures found from optical photometry and ionization equilibria and it is possible that similar differences will be present for the two methods used here. Hence, the temperature estimate for A04 should be treated with some caution.

Comparison of observed and theoretical Balmer lines (see Rolleston et al. 2000 for further details) were then used to estimate logarithmic surface gravities, which are again summarized in Table 3. Some of the surface gravity estimates differed significantly from the value (3.5 dex) assumed when initially estimating the effective temperatures. In these cases the effective temperatures were re-estimated using an appropriate gravity and the procedure iterated until it converged. An example of the quality of the fit can be found in Fig. 2 of Paper I and relevant uncertainties for the individual stars are also listed in Table 3.

For three stars (A02, B22, B37) there were sufficient $\mathrm{O}_{\text {II }}$ lines to estimate microturbulent velocities and these are again summarized in Table 3. For the other stars a value of $5 \mathrm{~km} \mathrm{~s}^{-1}$ was adopted; we note that although this may be too low, the weakness of the metal line spectra in the SMC targets implies that the choice of this parameter does not significantly affect the abundance analysis.

For the galactic standard, $\mathrm{BD}+56576$, the atmospheric parameters have been re-estimated using the same criteria and methods as for the NGC 330 targets and are summarized Table 4, together with the values deduced by Vrancken et al. (2000, VDLL). Given the different criteria and non-LTE methods used by VDLL, the agreement is surprisingly good. However, we emphasize that the values deduced here should not be considered as the best available but rather appropriate for a differential analysis with respect to the NGC 330 targets. The comparison with VDLL also provides us with an estimate of the importance of non-LTE effects which will be discussed in the next section.

In the case of AV304, we have adopted the atmospheric parameters (listed in Table 4) deduced by Rolleston et al. (2002). They used similar model atmosphere techniques and criteria to those adopted here, apart from the effective temperature which was estimated from the silicon ionization equilibria. However, in a previously analysis of this star (Rolleston et al. 1993) good agreement was found between the effective temperatures estimates found from the ionization equilibrium and the IUE flux distribution.

\section{Results}

Using the atmospheric parameters discussed above, the metal lines in the NGC 330 targets were used to deduce absolute 
Table 3. Atmospheric parameters (effective temperatures (K), logarithmic surface gravities (cgs), microturbulent velocities $\left(\mathrm{km} \mathrm{s}^{-1}\right)$ ) and LTE abundances for NGC 330 targets (the numbers in parentheses give the number of lines used to derive the mean abundance). For stars without an error estimate for the microturbulent velocity we have assumed a value of $5 \mathrm{~km} \mathrm{~s}^{-1}$.

\begin{tabular}{lllllllll}
\hline \hline Star & A01 & A02 & A04 & B04 & B22 & B32 & B37 & Mean \\
\hline$T_{\text {eff }}$ & 24000 & 16000 & 18000 & 23000 & 20000 & 22000 & 18000 & \\
$\log g$ & $3.7 \pm 0.3$ & $2.3 \pm 0.1$ & $2.8 \pm 0.2$ & $3.6 \pm 0.2$ & $3.0 \pm 0.2$ & $3.0 \pm 0.3$ & $2.4 \pm 0.1$ & \\
$v_{\mathrm{t}}$ & 5 & $8 \pm 4$ & 5 & 5 & $11 \pm 4$ & 5 & $10 \pm 4$ & \\
& & & & & & & & \\
He I & $10.78(10)$ & $10.58(10)$ & $10.66(9)$ & $10.78(10)$ & $10.82(10)$ & $10.85(8)$ & $10.98(10)$ & $10.78 \pm 0.13$ \\
C II & $6.82(1)$ & $6.91(2)$ & $7.05(2)$ & $6.86(1)$ & $6.89(2)$ & $6.54(1)$ & $6.71(2)$ & $6.85 \pm 0.15$ \\
N II & $7.62(8)$ & $7.71(9)$ & $7.16(2)$ & $7.54(7)$ & $7.52(7)$ & $7.40(3)$ & $7.89(9)$ & $7.62 \pm 0.18$ \\
O II & $8.13(16)$ & $8.14(12)$ & $8.23(2)$ & $7.88(5)$ & $7.83(7)$ & $8.23(3)$ & $7.98(12)$ & $8.05 \pm 0.13$ \\
Mg II & $6.78(1)$ & $6.69(1)$ & $6.43(1)$ & $6.68(1)$ & $6.54(1)$ & - & $6.79(1)$ & $6.65 \pm 0.14$ \\
Si II & - & $6.65(2)$ & $6.26(2)$ & - & - & - & & $6.5:$ \\
Si III & $6.75(3)$ & $6.82(2)$ & $6.08(2)$ & $6.55(3)$ & $6.49(3)$ & $6.25(1)$ & $7.12(3)$ & $6.63 \pm 0.32$ \\
Si IV & $7.19(1)$ & - & - & - & - & - & - & $7.2:$ \\
\hline
\end{tabular}

Table 4. Model atmosphere analysis of galactic standard, BD +56576 , together with the previous results of Vrancken et al. (2000 VDLL). The results for AV304 are taken directly from Rolleston et al. (2002) Numbers in parentheses refer to the number of lines used to derive the abundance.

\begin{tabular}{llll}
\hline \hline & This paper & VDLL & AV304 \\
Method & LTE & non-LTE & LTE \\
\hline$T_{\text {eff }}$ & 22000 & 22500 & 27500 \\
$\log g$ & 3.4 & 3.4 & 3.8 \\
$v_{\mathrm{t}}$ & 15 & 13 & 5 \\
& & & \\
$\mathrm{He}$ & $10.85(7)$ & - & 10.86 \\
$\mathrm{C}$ & $7.53(2)$ & 7.89 & 7.20 \\
$\mathrm{~N}$ & $7.41(7)$ & 7.35 & 6.66 \\
$\mathrm{O}$ & $8.66(16)$ & 8.57 & 8.23 \\
$\mathrm{Mg}$ & $7.13(1)$ & 7.08 & 6.79 \\
$\mathrm{Si}$ & $7.04(6)$ & 7.09 & 6.79 \\
\hline
\end{tabular}

abundances using atomic data taken from Jeffery (1991). These absolute values are listed in Table 3, together with the number of lines used in the analysis. Also listed for each ionization stage are the mean abundance estimate and the standard deviation. In Table 4, absolute abundance estimates are given for BD +56 576 (both deduced here and by Vrancken et al. 2000) and for AV304, which are again taken directly from Rolleston et al. (2002).

It is more useful to consider a differential analysis of the NGC 330 targets relative to the galactic standard BD 56576. This will be less affected by any errors in oscillator strengths or systematic errors in the adopted atmospheric parameters. This is particulary crucial for the relative carbon abundance due to the fact that the $4267 \AA$ line gives very different results from the 3919/3921 A doublet. Unfortunately, it was not possible to consider all the lines observed in the Caspec spectra due to the more limited wavelength coverage for BD +56576 . The differential abundances are summarized in Table 5 for each star, together with the means (and standard deviations) for each element. The results of a similar differential comparison for AV304 (relative to the galactic star HR 2387) taken from Rolleston et al. (2002) are also summarized in Table 5. These differential analyses confirm the main results from the absolute analyses - all the SMC targets are deficient in metals apart from nitrogen, which is very deficient in AV304 but slightly enhanced relative to our Galaxy in the NGC 330 targets. Below the results for individual elements are discussed in more detail.

\subsection{Helium}

An effectively normal helium abundance is found, although there is considerable scatter within the NGC 330 stars. This probably reflects the difficulty in estimating the continuum placement for the relatively broad diffuse helium lines, which make up the majority of our dataset. Hence we conclude that within the uncertainties, there is no evidence for an anomalous helium abundance in our targets.

\subsection{Carbon}

The differential abundances are based mainly on the $\mathrm{C}_{\text {II }}$ doublet at $4267 \AA$, supplemented in some cases by the line at $3921 \AA$. The former is know to be particularly sensitive to non-LTE effects (Eber \& Butler 1988). Also as discussed in Paper I the stellar absorption line could be affected by recombination from an associated HII region. However all the NGC 330 targets show a significant carbon underabundance (with a relatively small scatter between stars), while the mean differential abundance is similar to that found for AV304.

The latter result appears inconsistent with the results in Tables 2 and 3, where the corresponding abundances differ by 0.35 dex. However the value for AV304 was based on a mixture of $\mathrm{C}_{\text {II }}$ and $\mathrm{C}_{\text {III }}$ lines and if only the former are considered the difference in absolute abundances decreases to approximately 0.15 dex. The small remaining discrepancy of approximately 0.1 dex with the differential abundances arises from the different set of lines used in the two analyses.

\subsection{Nitrogen}

As discussed in Paper I, the most striking difference between the two NGC 330 targets and AV304 is in their N II spectra. 


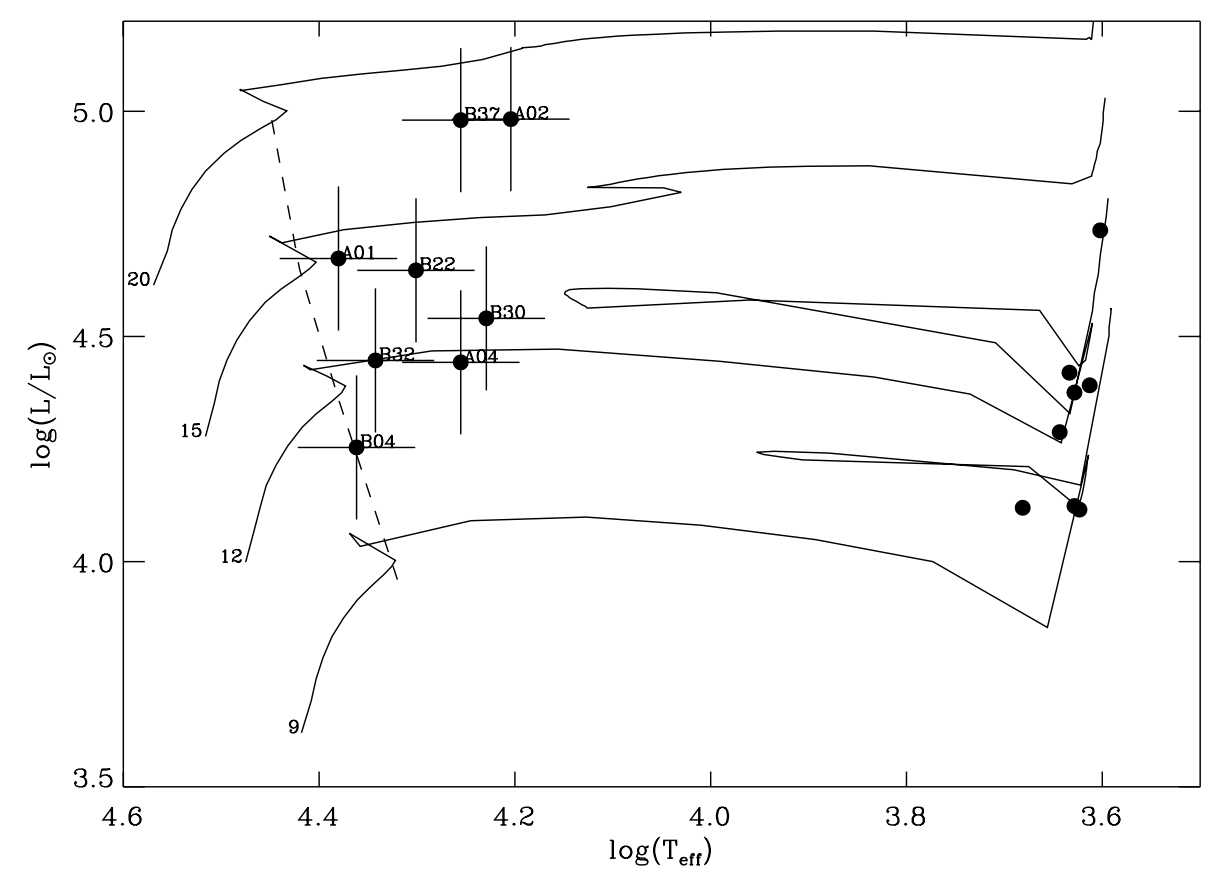

Fig. 2. HR-diagram for stars in NGC 330 showing the positions of the stars relative to the stellar evolution tracks (solid lines) of Charbonnel et al. (1993) which are computed for a metallicity of $Z=0.004$. Tracks are labeled with their initial masses. The dashed line represents the approximate position of the end of the core H-burning main sequence for the rotating models of Maeder \& Meynet (2001) for an assumed initial rotational velocity of $300 \mathrm{~km} \mathrm{~s}^{-1}$. Blue stars (analysed here) are labeled with their identifications. For comparison we also show the positions of the red supergiants in NGC 330 analysed by Hill (1999) and Gonzalez \& Wallerstein (1999), whose chemical compositions we also discuss. The error bars represent typical uncertainties discussed in the text, for example $15 \%$ in effective temperature.

Table 5. Differential abundance analyses of the NGC 330 targets and of AV304 relative to galactic standards BD +56576 and HR2387 respectively. The number of lines $(n)$ used in the derivation of these values is also given.

\begin{tabular}{|c|c|c|c|c|c|c|c|c|c|c|c|c|}
\hline \multirow[t]{2}{*}{ Star } & \multicolumn{2}{|l|}{$\mathrm{He} \mathrm{I}$} & \multicolumn{2}{|l|}{ C II } & \multicolumn{2}{|l|}{ N II } & \multicolumn{2}{|l|}{ O II } & \multicolumn{2}{|l|}{ Mg II } & \multicolumn{2}{|l|}{ Si III } \\
\hline & $\Delta\left[\frac{\mathrm{He}}{\mathrm{H}}\right]$ & $n$ & $\Delta\left[\frac{\mathrm{C}}{\mathrm{H}}\right]$ & $n$ & $\Delta\left[\frac{\mathrm{N}}{\mathrm{H}}\right]$ & $n$ & $\Delta\left[\frac{\mathrm{O}}{\mathrm{H}}\right]$ & $n$ & $\Delta\left[\frac{\mathrm{Mg}}{\mathrm{H}}\right]$ & $n$ & $\Delta\left[\frac{\mathrm{Si}}{\mathrm{H}}\right]$ & $n$ \\
\hline A01 & $-0.01 \pm 0.08$ & 7 & -0.43 & 1 & $+0.17 \pm 0.24$ & 7 & $-0.53 \pm 0.24$ & 16 & -0.35 & 1 & $-0.46 \pm 0.30$ & 3 \\
\hline A02 & $-0.31 \pm 0.17$ & 7 & $-0.62 \pm 0.21$ & 2 & $+0.30 \pm 0.19$ & 6 & $-0.54 \pm 0.30$ & 12 & -0.44 & 1 & $-0.37 \pm 0.06$ & 2 \\
\hline A04 & $-0.19 \pm 0.18$ & 6 & $-0.49 \pm 0.30$ & 2 & $-0.19 \pm 0.21$ & 2 & $-0.44 \pm 0.18$ & 2 & -0.70 & 1 & $-1.12 \pm 0.13$ & 2 \\
\hline B04 & $-0.02 \pm 0.12$ & 7 & -0.39 & 1 & $+0.13 \pm 0.33$ & 7 & $-0.62 \pm 0.23$ & 5 & -0.45 & 1 & $-0.67 \pm 0.41$ & 3 \\
\hline B22 & $+0.07 \pm 0.12$ & 7 & $-0.64 \pm 0.17$ & 2 & $+0.06 \pm 0.18$ & 5 & $-0.85 \pm 0.39$ & 7 & -0.59 & 1 & $-0.73 \pm 0.23$ & 3 \\
\hline B32 & $-0.01 \pm 0.14$ & 5 & -0.71 & 1 & $-0.09 \pm 0.03$ & 2 & $-0.39 \pm 0.04$ & 3 & - & - & -0.94 & 1 \\
\hline B37 & $+0.17 \pm 0.11$ & 7 & $-0.83 \pm 0.25$ & 2 & $+0.39 \pm 0.27$ & 6 & $-0.64 \pm 0.21$ & 12 & -0.34 & 1 & $-0.09 \pm 0.22$ & 3 \\
\hline Mean & $-0.04 \pm 0.15$ & - & $-0.61 \pm 0.15$ & - & $+0.17 \pm 0.16$ & - & $-0.59 \pm 0.11$ & - & $-0.48 \pm 0.14$ & - & $-0.57 \pm 0.33$ & - \\
\hline AV304 & $-0.01 \pm 0.04$ & 3 & $-0.58 \pm 0.11$ & 2 & $-1.17 \pm 0.08$ & 2 & $-0.38 \pm 0.11$ & 68 & -0.52 & 1 & $-0.50 \pm 0.03$ & 2 \\
\hline
\end{tabular}

However the new VLT observations for AV304, now allows this difference to be quantified. Indeed the mean differential abundance implies that for the NGC 330 targets nitrogen is overabundant by a factor of approximately twenty compared with AV304. This is consistent with Paper I, where a lower limit on the nitrogen enrichment of a factor of six was deduced relative to the SMC. There is considerable scatter in the differential abundances deduced for individual stars. This is some-

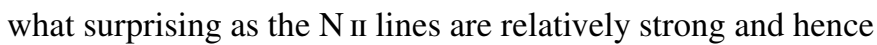
the equivalent width estimates should be reliable. Additionally, only small non-LTE effects have been found for the $\mathrm{N}$ II spectra (Becker \& Butler 1989). Hence the scatter could possibly reflect a real variation in the nitrogen abundances in these stars.

\subsection{Oxygen}

The $\mathrm{O}_{\text {II }}$ spectrum, together with that for $\mathrm{N}_{\text {II }}$ is the best observed in our targets. The differential oxygen abundances are in good agreement with only that for B22 being far from the mean. This star has a relatively low effective temperature estimate of $20000 \mathrm{~K}$ and a change of $1000 \mathrm{~K}$ in this estimate would change the differential abundance by more than 0.3 dex. Hence we believe that there is no evidence for any variation in the oxygen abundances among the NGC 330 targets. Additionally the mean differential abundance is similar to, although somewhat lower than, that found for AV304. 


\subsection{Magnesium}

Although the results are based solely on the $\mathrm{Mg}$ II doublet at $4481 \AA$, there is reasonable agreement in the individual differential abundances, whilst the mean value agree well with that for AV304.

\subsection{Silicon}

In contrast with oxygen and magnesium, the differential silicon abundances show a wide spread, which is characterised by the relatively large standard deviation in the mean value. The reason for this is unclear as for most of the targets the Si III lines near $4560 \AA$ are relatively well observed, although the relative strengths of these lines are sometimes anomalous. Additionally for the cooler targets, the estimated abundances are very temperature sensitive; for example at an effective temperature of $18000 \mathrm{~K}$, a shift in temperature of $1000 \mathrm{~K}$ changes the abundance by more than 0.3 dex. However the $\mathrm{O}_{\mathrm{II}}$ ion has a similar temperature sensitivity in this regime, whilst the oxygen abundance estimates are far more consistent. Although the cause of the discrepancies is unclear, they may well be due (at least in part) to the small number of measurable silicon lines. Indeed they are unlikely to reflect real abundance differences given the behaviour of other $\alpha$-process elements. The mean differential abundance is within the uncertainties in good agreement with that found for AV304.

\section{Discussion}

\subsection{The chemical composition of our B-type sample in NGC 330}

The principal conclusion from the differential analysis is that the cluster targets have a much higher nitrogen and possibly lower oxygen abundance than AV304. Relative nitrogen to oxygen abundances, $\left[\frac{\mathrm{N}}{\mathrm{O}}\right]$ of $-0.40 \pm 0.24$ dex and $-1.6 \mathrm{dex}$ are deduced for the NGC 330 targets and AV304 respectively. For the former the estimates from the individual stars have been weighted by the number of lines observed (although if the stars are uniformly weighted the ratio is only changed by $0.1 \mathrm{dex}$ ), whilst for the latter the uncertainty in the individual element abundances imply an error in the ratio of approximately 0.2 dex. For the NGC 330 targets, if the errors in the ratios are randomly distributed, the error on the mean would be reduced to approximately \pm 0.1 dex.

As discussed in Paper I, the theoretical $\mathrm{N}_{\text {II }}$ and $\mathrm{O}_{\text {II }}$ line strengths have a similar dependence on the adopted atmospheric parameters and hence the estimated nitrogen to oxygen abundance ratios are unlikely to be affected by uncertainties in these quantities. Hence we conclude that the $\left[\frac{\mathrm{N}}{\mathrm{O}}\right]$ ratio is enhanced by 1.2 dex with respect to AV304 and this estimate is unlikely to be significantly affected by uncertainties in atmospheric parameters or the relatively simple LTE analysis adopted.

The simplest explanation for this nitrogen enhancement is that it represents the products of hydrogen burning by the CNO bi-cycle. In such circumstances, it might be expected that there should be a corresponding enhancement in helium and underabundances in carbon and possibly oxygen, with the sum of CNO nuclei in the NGC 330 stars comparable to that in AV304. It is therefore important to try to map the abundances for the NGC 330 targets as derived from our differential analysis onto an absolute scale. There are a number of options available to us, which we now discuss.

If we assume that the composition of AV304 is the baseline initial composition of NGC 330, then we can use the difference in the LTE abundances of the NGC 330 targets relative to AV304. However we must also address the probable impact of non-LTE (NLTE) effects on these abundances. We have used NLTE calculations similar to those discussed in McErlean et al. (2000) to estimate the difference in NLTE and LTE CNO abundance estimates for AV304. The corrections are approximately $+0.07,-0.11$ and -0.07 dex respectively for $\mathrm{C}, \mathrm{N}$ and $\mathrm{O}$. In addition we note that our carbon abundance in AV304 relies heavily on the $4267 \AA$ line which is well known to give spuriously low abundances. Following the discussion of Vrancken et al. (2000) and comparing their results with those of Gies \& Lambert (1992) we further correct the carbon abundance by +0.34 dex. This corresponds to the difference between their respective NLTE results which reflects their different selections of absorption lines, Vrancken et al's results depending largely on the 4267 and 3919/21 $\AA$ doublets, as is the case in the present paper. However given the magnitude of the correction for carbon, we regard the final values as more uncertain than those for nitrogen and oxygen. Our final CNO NLTE abundances estimates for AV304 are then 7.41, 6.55 and 8.16 dex in good agreement with the $\mathrm{H}$ iI region results of 7.4, 6.6 and 8.1 dex as summarized in the discussion of baseline SMC abundances for A-type supergiants in the SMC by Venn (1999).

We can now use these modified NLTE abundance estimates for AV304 and the difference in LTE abundances listed in Tables 3 and 4 to estimate absolute CNO abundance for our NGC 330 targets; these are summarized in Table 6. In turn we can then estimate the sum of the $\mathrm{CNO}$ and $\mathrm{CN}$ abundances for AV304, which are 8.24 and 7.46 dex respectively. These may be compared with the mean NGC 330 totals of 8.17 and 7.71 dex respectively. There is some slight evidence for an increase in $\mathrm{CN}$ but the difference of +0.25 should be compared with uncertainties in the mean carbon and nitrogen abundances of 0.15 and 0.18 dex (the total being dominated by the more abundant species). The sum of CNO is in good agreement but again the total is dominated by the oxygen abundance for which the uncertainty is approximately 0.13 dex. Clearly it is unproductive to compare summations of abundances when one species is substantially more abundant than all other species, and the uncertainty in that abundance is similar to, or larger than, that of the less abundant species.

We arrive at a similar picture if we instead consider the differential abundances of the NGC 330 stars relative to BD +56576 using the NLTE abundances published by Vrancken et al. (2000) to put them on an absolute scale. This results in CNO abundances for the NGC 330 targets of 7.28, 7.52 and 7.98 dex respectively which are in good agreement with the values obtained using AV304 as the standard (see Table 6). 
Table 6. Comparison of our abundance estimates corrected for NLTE effects with results for other stars in NGC 330, viz. the B-type star B30 analysed by Korn et al. (NLTE C and O) and Reitermann et al. (LTE N) (B30) and cool supergiants from Hill (H99) and Gonzalez \& Wallerstein (GW). Also tabulated are other SMC abundance estimates, viz. the main sequence star AV304 (Rolleston et al. 2002 corrected for NLTE effects), A-type supergiants from Venn (A-stars), B-type giants and supergiants from Korn et al. (B-stars) and cool supergiants from Hill et al. (K-stars).

\begin{tabular}{llllllllll}
\hline \hline & & \multicolumn{9}{c}{ NGC 330 } & & \multicolumn{3}{c}{ SMC supergiants } \\
Element & AV304 & This paper & B30 & H99 & GW & & A-stars & B-stars & K-stars \\
\hline $\mathrm{C}$ & 7.47 & 7.26 & 7.3 & 7.40 & 7.55 & & - & 7.40 & 7.55 \\
$\mathrm{~N}$ & 6.55 & 7.52 & 7.4 & 7.21 & 6.96 & & 7.33 & 7.25 & 7.51 \\
$\mathrm{O}$ & 8.16 & 7.98 & 8.25 & 7.71 & 7.92 & & 8.14 & 8.15 & 8.01 \\
\hline
\end{tabular}

This is possibly fortuitous, and given the difficulty in modeling the $4267 \AA$ line, unexpected for carbon. Nevertheless it reinforces the previous discussion of the absolute abundances. We conclude that the chemical peculiarities of the NGC 330 targets may be understood in terms of nuclear processing by the $\mathrm{CNO}$ bi-cycle, perhaps with some weak evidence that ON processing has occurred, but it is not necessary to invoke primary nitrogen production (although this cannot be precluded).

We can also search for correlations between element abundances within the NGC 330 targets. Linear least squares fits show a positive correlation between the helium and nitrogen abundances and negative correlations between the carbon and nitrogen and between the oxygen and nitrogen abundances. Interestingly all these trends are consistent with the transformation of hydrogen into helium using the $\mathrm{CNO}$ bicycle. Unfortunately however, none of the correlations are convincing and the coefficients are not significantly different from zero at even the $1 \sigma$ level.

\subsection{Other stellar abundances in NGC 330 and the SMC}

Korn et al. (2000) have recently published C, O, Mg and $\mathrm{Si}$ NLTE abundances for another B-type giant in NGC 330, the star B30, and their abundances are in good agreement with ours given the magnitude of the NLTE corrections and the uncertainties in both studies. Unfortunately they do not give a nitrogen abundance but two other similar SMC giants in their sample have NLTE nitrogen abundances of 7.3 and 7.2 dex. We will return to these stars in the discussion of their evolutionary status below. Furthermore an LTE nitrogen abundance for star B30 was published by Reitermann et al. (1990) who obtained a value of $7.4 \mathrm{dex}$ for a microtubulence of $5 \mathrm{~km} \mathrm{~s}^{-1}$.

There have also been many studies of cool giants and supergiants in NGC 330. These results are summarized in Table 6. Of interest here are $\mathrm{CNO}$ abundances and there are two recent estimates for NGC 330 stars by Hill (1999, H99) and Gonzalez \& Wallerstein (1999, GW). One obvious difference between these is that the mean nitrogen abundance of $\mathrm{GW}$ is systematically lower than that of H99. However we note that the nitrogen abundance is derived from molecular $\mathrm{CN}$ features, and depends on the adopted carbon abundance (which is derived from $\mathrm{C}_{2}$ ). While $\mathrm{H} 99$ independently derive their carbon abundances, GW adopted mean values from the literature and there is a small systemic overestimation relative to H99 (approximately 0.2 dex). Such a small change in the carbon abundance is the most likely reason for their low nitrogen abundances and given that the carbon abundance of H99 may be more reliable and that their results agree better with other samples of evolved B, A and F-type stars in the SMC, we prefer their results for carbon and nitrogen.

Comparing with other SMC samples we note that our mean CNO abundances are in good agreement with the results of Venn (1999) and Hill et al. (1997) although the NGC 330 stars may be mildly metal poor. There have been previous suggestions that NGC 330 may be relatively metal poor with respect to the SMC (Grebel \& Richtler 1992) but our results confirm recent work in that any metal deficiency must be small $(<0.2$ dex $)$. We therefore conclude that the pattern of the mean CNO abundances found in the NGC 330 B-type giants is very similar to that found for samples of other evolved A, $\mathrm{F}$ and K-type giants and supergiants. We also note that Dufton et al. (2000) investigated a large sample of B-type supergiants in the SMC and the nitrogen abundances found in their less luminous stars, although uncertain, are comparable to those found here.

\subsection{Evolutionary status}

It is useful to preface our discussion of the evolutionary status of these stars by considering their positions in the HR-diagram. We follow the procedure described in VDLL, adopting a distance modulus for the SMC of 18.9 and extinction estimates as discussed in Sect. 3.1. As in VDLL we estimate spectroscopic masses $\left(M_{\mathrm{spec}}\right)$ from our derived stellar radii and surface gravities. Figure 2 illustrates the positions of the stars compared with the non-rotating stellar evolution tracks of Charbonnel et al. (1993) which are computed with metallicity of $Z=0.004$ (SMC-like). Again following VDLL we estimate evolutionary masses $\left(M_{\text {evol }}\right)$ by interpolation in this diagram and these may be compared with $M_{\text {spec }}$ in Table 7. where all derived quantities are summarized. We note that Maeder \& Meynet (2001) have produced a grid of calculations including the effects of rotation for a metallicity of $Z=0.004$. Figure 2 also shows the locus of the end of H-burning main sequence phase for an assumed initial rotational velocity of $300 \mathrm{~km} \mathrm{~s}^{-1}$ (taken from their Fig. 6). Note that this locus also corresponds to the approximate position of the end of the main sequence for the non-rotating models. This is a consequence of the fact that the main sequence widening in Charbonnel et al. comes from their inclusion of convective overshooting, while a similar effect is obtained by 
Table 7. Derived quantities for B-type stars in NGC 330: Stellar radii $\left(R / R_{\odot}\right)$, absolute visual magnitudes $\left(M_{\mathrm{v}}\right)$, bolometric corrections $(B . C$.), bolometric magnitudes $\left(M_{\mathrm{bol}}\right)$, luminosities $\left(\log \left(L / L_{\odot}\right)\right.$ and estimates of spectroscopic $\left(M_{\text {spec }}\right)$ and evolutionary $\left(M_{\text {evol }}\right)$ masses in units of solar mass. The distance modulus to the SMC was assumed to be 18.9 in the derivation of these quantities. The last two columns compare the difference in spectroscopic and evolutionary masses with the uncertainties in $\log g(\Delta \log g)$.

\begin{tabular}{lrllllrrrc}
\hline \hline Star & $R / R_{\odot}$ & $M_{\mathrm{v}}$ & B.C. & $M_{\text {bol }}$ & $\log \left(L / L_{\odot}\right)$ & $M_{\text {spec }}$ & $M_{\text {evol }}$ & $\log \left(M_{\text {evol }} / M_{\text {spec }}\right)$ & $\Delta \log g$ \\
\hline A01 & 12.6 & -4.48 & -2.50 & -6.98 & 4.67 & 29 & 15 & -0.29 & 0.3 \\
A02 & 40.4 & -6.28 & -1.49 & -7.77 & 4.98 & 12 & 18 & 0.18 & 0.1 \\
A04 & 17.2 & -4.64 & -1.77 & -6.41 & 4.44 & 7 & 12 & 0.23 & 0.2 \\
B04 & 8.5 & -3.58 & -2.34 & -5.92 & 4.25 & 10 & 11 & 0.04 & 0.2 \\
B22 & 17.6 & -4.89 & -2.03 & -6.92 & 4.65 & 11 & 14 & 0.10 & 0.2 \\
B32 & 11.5 & -4.17 & -2.23 & -6.40 & 4.45 & 5 & 12 & 0.38 & 0.3 \\
B37 & 31.9 & -5.99 & -1.76 & -7.75 & 4.98 & 9 & 18 & 0.30 & 0.1 \\
B30 & 21.6 & -5.08 & -1.64 & -6.72 & 4.54 & 11 & 13 & 0.07 & 0.2 \\
\hline
\end{tabular}

Maeder \& Meynet with rotationally induced mixing alone. The fact that overshooting and rotation both have similar results in this respect has been discussed previously, see for example Talon et al. (1997). We will return to this point later in the discussion, after first considering the observed abundance pattern.

Nitrogen enrichment is clearly a sign of contamination of the surface by the products on the nuclear burning, in this case from the CNO bi-cycle. What is especially interesting about the current sample of stars (to which we can add the star B30 mentioned above) is that they represent a coeval group of stars with rather homogeneous properties, whose N/C surface abundance ratio is enhanced by typically a factor of 10 . Stellar models which include the effects of rotationally induced mixing have been proposed as a means of producing the observed nitrogen enhancements in main sequence and evolved massive stars. Maeder \& Meynet (2001) have followed the evolution of the surface abundances for a range of initial rotational velocities. Comparing our results to the models with high initial rotational velocity, $v_{\text {ini }}=300 \mathrm{~km} \mathrm{~s}^{-1}$, and therefore relatively large nitrogen enhancements, we find that the best agreement is with their red supergiant or blue loop stars. At the end of the main sequence these models predict an increase in $[\mathrm{N} / \mathrm{H}]$ by a factor of only 3 , or about 0.5 dex. Note however that their initial abundance ratios are assumed to be solar and therefore they overestimate the initial nitrogen abundance significantly, adopting one fifth solar, which is approximately $7.3 \mathrm{dex}$ in our notation.

We can perform a simple recalibration of their results by assuming that a calculation with an initial lower nitrogen abundance of $6.6 \mathrm{dex}$ (but similar carbon and oxygen) produces the same excess of nitrogen in absolute terms. In this case the nitrogen abundance at the end of the main sequence would be approximately 7.7 dex rather than $7.8 \mathrm{dex}$, a consequence of the fact that the initial nitrogen abundance is negligible compared with that which is produced by the star. These models therefore could conceivably reproduce the observed nitrogen enhancements. In fact if the initial $\mathrm{N} / \mathrm{O}$ or $\mathrm{N} /(\mathrm{O}+\mathrm{C})$ abundance ratios are small and can be neglected, which in the case of the SMC is a good approximation, then it is easy to show that one only needs a relatively small fraction of core material in ON equilibrium to produce a big change in the observed nitrogen abundance.
Nevertheless, as Fig. 2 shows, there are other more serious discrepancies. For example, our objects tend to lie red-wards of the main sequence despite the widening provided by the rotating models (and models with convective overshooting). In addition all the B-type giants/supergiants in our sample are slow rotators with values of $v \sin i$ less than $50 \mathrm{~km} \mathrm{~s}^{-1}$. By contrast the rotating models require very high initial rotation to produce the enhanced nitrogen but do not predict significant slow down by the end of the main sequence. As discussed in Paper I, while there may be a selection effect in our observed sample (we can only analyse the slow rotators) it is highly unlikely that there are so many fast rotators in NGC 330 oriented pole on. For example, both Mazzali et al. (1994) and Keller \& Bessell (1998) give $v \sin i$ values for 22 of the brightest $\mathrm{B}$ and Be-type stars in NGC 330. They find that maximum values lie in the range $300-400 \mathrm{~km} \mathrm{~s}^{-1}$ and if we assume that our sample have similar rotation rates $(v)$ this implies that $\sin i$ is less than 10 degrees. In other words if the distribution of $i$ is random we should expect our sample to be drawn from about $1.5 \%$ of all the B-type stars in NGC 330. This is clearly incompatible with the number of B-type stars in NGC 330 in the relevant magnitude range. One is left with the conclusion that our objects are intrinsically slow rotators at the present time. Either they were fast rotators in the past, and have somehow slowed down, or some process other than rotationally induced mixing is responsible for the observed abundance pattern.

Given the similarity between the carbon and nitrogen abundances in the blue and red stars in NGC 330 it is tempting to invoke blue loops as a means of explaining our abundances. This seems unlikely given that no stellar evolution calculations predict loops which progress hotter than effective temperatures corresponding to late-B spectral types. While Venn (1999) invoked blue loops for the A-type supergiants this does not seem a viable option for our early B-type stars, despite similarities in CNO abundances. Mass-transfer in binaries may also be invoked to explain enhanced nitrogen abundances and Wellstein et al. (2001) have recently produced models which produce nitrogen enriched blue stars which can reside in the post mainsequence gap. Such stars may also appear to be under-massive for their luminosities and is therefore tempting to ascribe the discrepancies between spectroscopic and evolutionary masses in Table 7 to binarity. One should be cautious however because 
our spectroscopic masses were estimated from the derived surface gravities and in some cases the uncertainties in this quantity are quite substantial. The final two columns of Table 7 compares the differences between spectroscopic and evolutionary masses with the uncertainties in gravity and in all but three cases (A02, B32 and B37) the mass differences are easily accounted for. There does appear to be a suggestion of a correlation between nitrogen enhancement and luminosity in our sample. Stars A02 and B37 are the most luminous and most N-rich of our sample, however their positions are perhaps consistent with being core-helium burning stars on their way red-wards (this phase represented by the slight kink in the 20 solar mass track for the non-rotating models). The problem for the rotating models is that this phase becomes progressively cooler and shorter lived as initial rotational velocity is increased, with only the slow rotators spending any significant time in this part of HR-diagram. However, such stars should not be significantly $\mathrm{N}$-enriched, in contradiction to the observations. B32 would appear to be the best candidate for the binary evolution hypothesis, the real problem being that much better constraints are needed on the gravity of this object, and indeed all other stars in our sample, before definitive statements can be made about possible mass discrepancies. Finally, while binarity appears to be an attractive scenario, it has a significant problem in that it is expected that the products of mass accretion will be fast rotators having been spun up by the accreted material. In addition radial velocities of all our stars are typical of the NGC 330 cluster (Table 1) although it must be noted that expected radial velocity amplitudes of the kind of systems predicted by the models of Wellstein \& Langer (2001) are typically the order of $10-20 \mathrm{~km} \mathrm{~s}^{-1}$.

Finally we note that VDLL carried out a similar study to the present one but for the solar metallicity cluster $\mathrm{h}+\chi$ Per. They did not find evidence for significant nitrogen overabundances in any of the evolved B-type stars in this cluster. However if we take the nitrogen enrichments found here, in absolute terms, apply these to their galactic counterparts it is clear that this leads to enhancements the order of a factor of 2-3. For some objects in $\mathrm{h}+\chi$ Per this magnitude of a nitrogen enhancement is consistent with the observations. It is simply more obvious in the SMC stars given their initial very low nitrogen abundance.

\section{Summary}

In this paper we have presented new results for 5 B-type giants/supergiants in the SMC cluster NGC 330. Together with our previous work, plus results for one other such star in NGC 330 presented by Korn et al. this brings the total of bright B-type stars analysed in this cluster to 8 . All the stars have the following characteristics:

- They are nitrogen rich and possibly carbon poor, with perhaps some marginal evidence for oxygen depletion, with their $N / C$ ratios being enhanced by approximately a factor of 10 relative to the SMC norm. The nitrogen enhancements are remarkably homogeneous.

- They all lie close to the main-sequence but with a strong tendency to lie beyond the end of the main-sequence even if main-sequence widening through rotation or convective overshooting is considered.

- They all appear to be currently slow rotators with $v \sin i$ values below $50 \mathrm{~km} \mathrm{~s}^{-1}$. The sample is too large for this to be an inclination angle effect for fast rotators.

- The nitrogen enhancements agree well with those found for cool red supergiants in the same cluster.

Single star stellar evolution models including the effects of rotation may indeed be able to explain the abundance patterns, although we note that there is a clear need for stellar evolution calculations with the correct mix of abundances for the SMC, in particular scaling the solar composition produces much too high an initial nitrogen abundance for the SMC. However single star models suffer from severe difficulties in reproducing both the effective temperatures of our sample and their low rotational velocities. Binary star models may be able to produce stars in the correct effective temperature range but may also have similar problems to single star models in reproducing the low rotational velocities. The similarity between blue Btype giant/supergiant nitrogen enhancements and those found for red supergiants in NGC 330 is surprising since one might expect the latter to be more nitrogen enriched with the blue stars' enhancement coming from rotation while that of the red stars coming from dredge-up processes. In conclusion, the B-type stars considered here are still something of a puzzle and not well modeled by current stellar evolution calculations. Moreover, given the large number of these objects in NGC 330 it is clear that they are not the product of some peculiar evolutionary path and therefore represent an important challenge to current stellar evolution theory.

Acknowledgements. Data reduction was performed on the PPARC funded Northern Ireland staRLINK node. DJL is grateful for NOVA funding for a visit to Utrecht in May 2001 during which time much of the present work was carried out. Thanks are also due to a number of people for contributions to this project; Norbert Langer, Paolo Mazzali, Gianni Marconi, Robert Rolleston and Kim Venn.

\section{References}

Arp, H. 1959, AJ, 64, 254

Balona, L. A. 1992, MNRAS, 256, 425

Becker, S. R., \& Butler, K. 1989, A\&A, 209, 244

Caloi, V., Casstella, A., Castellani, V., \& Walker, A. 1993, A\&A, 271, 109

Carney, B. W., Janes, K. A., \& Flower, P. J. 1985, AJ, 90, 1196

Charbonnel, C., Meynet, G., Maeder, A., Schaller, G., \& Schaerer, D. 1993, A\&AS, 101, 415

Chiosi, C., Vallenari, A., Bressan, A., Deng, L., \& Ortolani, S. 1995, A\&A, 293, 710

Dufton, P. L., McErlean, N. D., Lennon, D. J., \& Ryans, R. S. I. 2000, A\&A, 353, 311

Eber, F., \& Butler, K. 1988, A\&A, 202, 153

Feast, M. W. 1972, MNRAS, 159, 113

Gies, D. R., \& Lambert, D. L. 1992, ApJ, 387, 673

Gonzalez, G., \& Wallerstein, G. 1999, ApJ, 117, 2286

Grebel, E. K., \& Richtler, T. 1992, A\&A, 254, 359

Grebel, E. K., James Roberts, W., \& Brandner, W. 1996, A\&A, 311, 470

Hill, V., Barbuy, B., \& Spite, M. 1997, A\&A, 323, 461 
Hill, V. 1999, A\&A, 345, 430

Jeffery, C. S. 1991, Newsletter on Analysis of Astronomical Spectra, No. 16,17

Keller, S. C., \& Bessell, M. S. 1998, A\&A, 340, 397

Keller, S. C., Bessell, M. S., \& Da Costa, G. S. 2000, AJ, 119, 1748

Kilian, J. 1994, A\&A, 262, 171

Kilian, J. 1994, A\&A, 282, 867

Korn, A. J., Becker, S. R., Gummersbach, C. A., \& Wolf, B. 2000, A\&A, 353, 655

Kurucz, R. L. 1992, Rev. Mex. Astrofis., 23, 45

Langer, N., \& Maeder, A. 1995, A\&A, 295, 685

Lennon, D. J., Mazzali, P. A., Pasian, F., Bonifacio, P., \& Castellani, V. 1993, Space Sci. Rev., 66, 169

Lennon, D. J., Dufton, P. L., Mazzali, P. A., Pasian, F., \& Marconi, G. 1996, A\&A, 314, 243

McErlean, N. D., Lennon, D. J., \& Dufton, P. L. 2000, A\&A, 349, 553

Mazzali, P. A., Pasian, F., Lennon, D. J., Bonifacio, P., \& Castellani, V. 1994, Proc. IAU Symp., 162, ed. L. Balona, H. Heinrichs, \& J. M. Le Contel (Kluwer, Dordrecht), 269

Maeder, A., Grebel, E., \& Mermilliod, J. C. 1999, A\&A, 346, 459

Maeder, A., \& Meynet, G. 2001, A\&A, 373, 555

Mazzali, P. A., Lennon, D. J., Pasian, F., et al. 1996, A\&A, 316, 173

Reitermann, A., Stahl, O., Wolf, B., \& Baschek, B. 1990, A\&A, 234, 109

Robertson, J. W. 1974, A\&AS, 15, 261
Rodriguez-Pascuel, P. M., Gonzalez-Riestra, R., Schartel, N., \& Wamsteker, W. 1999, A\&AS, 139, 183

Rolleston, W. R. J., Dufton, P. L., Fitzsimmons, A., Howarth, I. D., \& Irwin, M. J. 1993, A\&A, 277, 10

Rolleston, W. R. J., Venn, K. A., Tolstoy, E., \& Dufton, P. L. 2002, A\&A, submitted

Rolleston, W. R. J., Smartt, S. J., Dufton, P. L., \& Ryans, R. S. I. 2000, A\&A, 363, 537

Seaton, M. J. 1979, MNRAS, 187, 73

Smartt, S. J., Rolleston, W. R. J., \& Dufton, P. L. 1996, A\&A, 305, 164

Smartt, S. J., Venn, K. A., Dufton, P. L., Rolleston, W. R. J., \& Keenan, F. P. 2001, A\&A, 367, 86

Spite, F., Richtler, T., \& Spite, M. 1991, A\&A, 252, 557

Stothers, R. B., \& Chin, C.-W. 1992a, ApJ, 390, L33

Stothers, R. B., \& Chin, C.-W. 1992b, ApJ, 390, 136

Talon, S., Zahn, J.-P., Maeder, A., \& Meynet, G. 1997, A\&A, 322, 209

Thompson, G. I., Nandy, K., Morgan, D. H., \& Houziaux, L. 1988, MNRAS, 230, 429

Venn, K. A. 1999, ApJ, 518, 405

Vrancken, M., Lennon, D. J., Dufton, P. L., \& Lambert, D. L. 2000, A\&A, 358, 639

Wellstein, S., Langer, N., \& Braun, H. 2001, A\&A, 369, 939

Willmarth, D., \& Barnes, J. 1994, A user's guide to reducing echelle spectra with IRAF, NAOA (Tucson, Internal Document) 